\title{
Computational analysis of intermolecular interactions in a crystal with structural phase transitions
}

\author{
D. Takagi ${ }^{1}$, T. Asahi ${ }^{1,2}$, T. Taniguchi ${ }^{3}$ \\ ${ }^{1}$ Graduate School of Advanced Science and Engineering, Waseda University, 3-4-1 Okubo, Shinjuku-ku, Tokyo, Japan, \\ ${ }^{2}$ Reaearch Organization for Nano \& Life Innovation, Waseda University, 513 Waseda-tsurumakicho, Shinjuku-ku, Tokyo, Japan, \\ ${ }^{3}$ Center for Data Science, Waseda University, 1-6-1 Nishi-waseda, Shinjuku-ku, Tokyo, Japan
}

daichan.t@fuji.waseda.jp

Molecular crystals with structural phase transitions are expected as novel materials for actuators and sensors. The design of crystals with phase transitions has been challenged by experimental and theoretical approaches. However, the former approach requires huge time and cost for experiments, and the latter requires high computational accuracy due to the high degree of freedom of molecular conformation. In recent years, the inductive approach to obtain knowledge from known data has been attracting attention as materials informatics. In this study, the intermolecular interactions in the (S)-N-3,5-di-tert-butylsalicylidene-1-(1-naphthyl)ethylamine (enol-( $S$ )1) crystals were analyzed, and we found new insights on structural phase transitions.

The enol- $(S)-1$ crystal has three crystal phases, namely $\alpha\left(<-80^{\circ} \mathrm{C}\right), \beta\left(-80 \sim 40^{\circ} \mathrm{C}\right)$, and $\gamma\left(<40^{\circ} \mathrm{C}\right)$ phases, which are reversible through single-crystal-to-single-crystal phase transition depending on temperature change[1]. First, the lattice energies were calculated at each temperature point of the enol-(S)-1 crystals to elucidate the whole strength of intermolecular interactions. The lattice energies showed a discontinuous temperature dependence according to the phase transition, with a slight decrease at the phase transition from $\alpha$ to $\beta$ phase and then an increase with rising temperature (Fig.1a). Then, the analysis of intermolecular interactions by Hirshfeld surfaces and 2D fingerprint plots was performed to reveal which interaction has contribution on the phase transitions. The Hirshfeld analysis uncovered that the proportion of $\pi \cdots \pi$ interactions decreased while the proportion of $\mathrm{CH} \cdots \pi$ interactions increased at the phase transition from $\alpha$ to $\beta$ phase. In addition, while the proportion of $\pi \cdots \pi$ interaction did not change, the proportion of $\mathrm{CH} \cdots \pi$ interaction decreased in the $\beta$ phase as the temperature rose but increased slightly after the transition from the $\beta$ to $\gamma$ phase. As to interaction energies, it was shown that the intermolecular interaction of $\mathrm{CH} \cdots \pi$ stabilized at the transitions from $\alpha$ to $\beta$ phase and from $\beta$ to $\gamma$ phase (Fig.1b). $\mathrm{CH} \cdots \pi$ interactions had the unique temperature dependence compared to other main interactions of $\pi \cdots \pi$ and $\mathrm{CH} \cdots \mathrm{O}$ interactions. These results clarify the contribution of $\mathrm{CH} \cdots \pi$ interaction to the stability of the high-temperature crystal phases and may provide new insights for designing crystals with phase transitions.
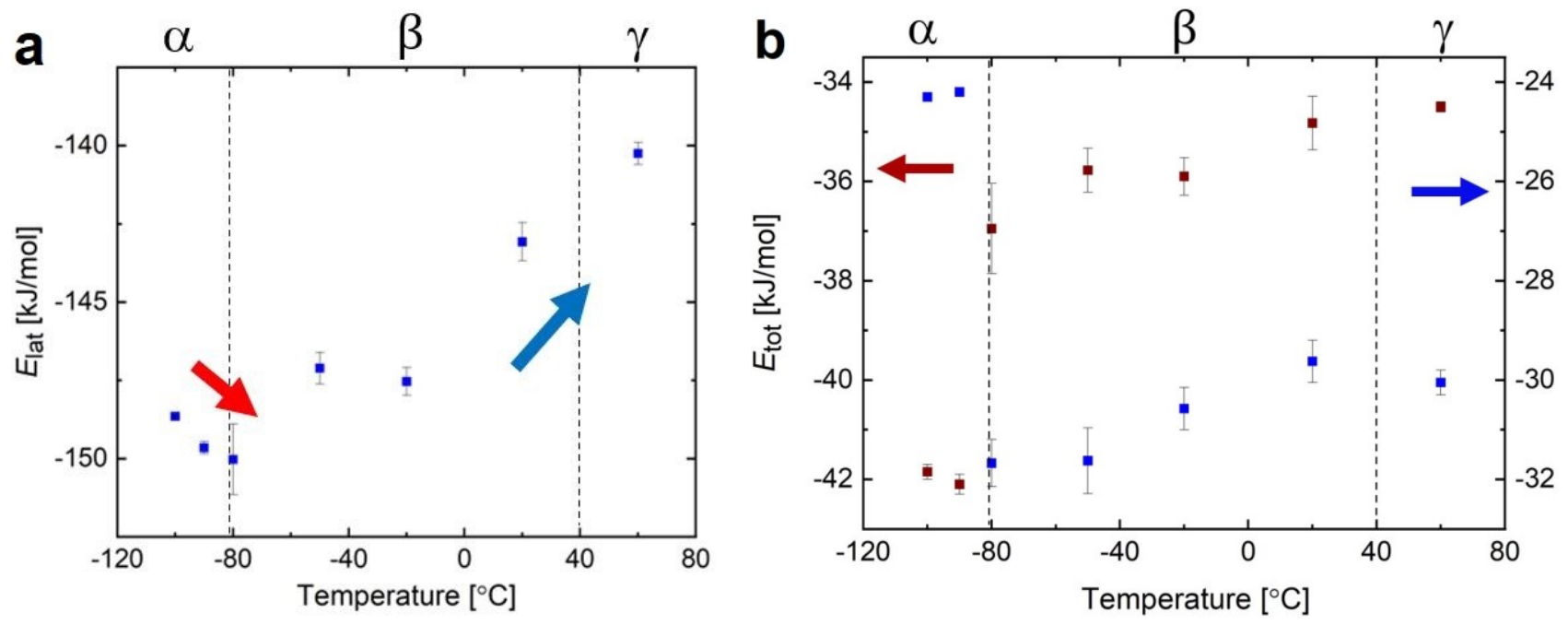

Figure 1. (a) Temperature dependence of lattice energies of enol-(S)-1 crystal.

(b) Temperature dependence of $\pi \cdots \pi$ (red) and $\mathrm{CH} \cdots \pi$ (blue) interaction energies.

[1] Taniguchi, T., Sato, H., Hagiwara, Y., Asahi, T., \& Koshima, H. (2019). Communications Chemistry, 2(1), 1-10.

Keywords: organic crystal; phase transition; intermolecular interaction energy; lattice energy; temperature dependence 